\title{
Semantic Grid based e-Learning using the Knowledge Life Cycle
}

\author{
Feng Tao, David Millard, Arouna Woukeu, Hugh Davis \\ University of Southampton \\ $\{f t$,dem,aw1,hcd\}@ecs.soton.ac.uk
}

\begin{abstract}
In this paper we suggest that if semantics are to fullfil their potential in the learning domain then a paradigm shift in perspective is necessry from information based content delivery to knowledge based, context-aware collaberative learning services. We propose a semantics driven knowledge life cycle that characterises the key phases in managing semantics and knowledge, and show how this can be applied to the learning domain.
\end{abstract}

\section{Introduction}

Both Web and Grid service architectures have been applied to the e-learning domain $[1,2]$, they are advantageous as they are modular and extensible and offer increased interoperability. The semantic aspects of learning content are the key to facilitating large scale collaboration of e-learning activities over service-oriented infrastructures.

An ontology is the best vehicle in this context to formally hold a specification (of the conceptualisation) that can be shared within the community to describe semantics accurately and consistently. It explicitly defines the domain concepts and their relationships and is similar to a dictionary or glossary, but with richer structure, relationship and axioms that describe a domain of interest more precisely.

These rich semantics offer both teachers and learners new oppertunities for locating and reusing resources $[3,4,5]$. But defining the correct semantics for a learning application is difficult, and maintaining ontologies can be problematic (akin to managing the evolution of a complex graph).

We propose a knowledge life cycle for learning, to help define and maintain evolving semantics [6]. Our intention is not to develop a definitive ontology, or to promote a particular architecture, but to demonstrate how a semanticdriven knowledge life cycle model can be applied to the learning domain.

\section{A Pedagogical View of Semantics}

Increased semantics offer students a more effective view of their learning and enables new learning opportunities $[3,5]$. There are a number of the ways in which reasoning about semantics can improve learning opportunities.

- Connecting Communities: Services can put people in contact with other people who are experts or learners with similar interests.

- Personalised Content: Intelligent tutoring systems have for some time being delivering content that was personalised for the user, based on an understanding of their goals and previous knowledge.

- Adaptive Assessment: Systems may choose questions for the learner at the boundary of their understanding, thus improving the efficiency of assessment and providing feedback that provides detail in critical areas.

- Annotation Tools: Users could annotate information themselves, providing useful information for others and allowing both readers and other services the opportunity to process the information in alternative ways.

E-Learning practitioners often comment that they believe they spend as much time organizing materials as they spend on teaching and the production of materials. We believe that semantics may ease these problems as well, e.g., by helping with time table management. We believe that appropriate semantic enrichment of the elements in the learning domain should make possible the automatic creation of workflows by the composition of appropriate services.

To fully realise the potential of semantics in the pedagogical domain (as described above) it is necessary to move from Metadata to Ontologies and from Information to Knowledge. Ontologies are a more sophisticated way of modelling metadata, and knowledge is relevant information delivered at the right time and context.

\section{The Knowledge Life Cycle}

The Knowledge Life Cycle is a model that describes how knowledge is captured, applied and reused. Figure 1 shows the four main phases of the Knowledge Life Cycle:

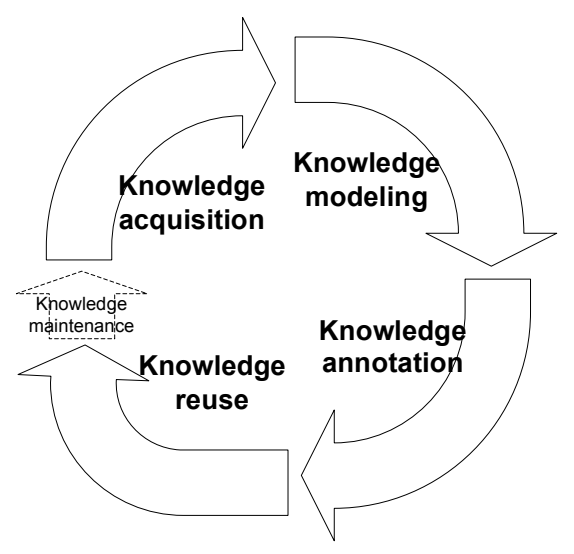

Figure 1: The Knowledge Life Cycle 
- Knowledge Acquisition (KA): Acquire the knowledge from domain experts (e.g. scenario construction and interviews) to develop domain vocabulary and a sense of important concepts.

- Knowledge Modelling (KM): The description is formalised as an ontology. Classes are defined and relationships between those classes are specified.

- Knowledge Annotation: The ontology is tested through application. Example resources from the domain are annotated with ontological metadata.

- Knowledge Reuse: New applications reuse the resources (made possible by the shared ontology), e.g. using learning objects in a new course design.

\section{Technical View of Learning Semantics}

In this section we describe how we have used the knowledge life cycle to develop reusable semantics for learning. The first part of the Knowledge Life Cycle is the process of Knowledge Acquisition. In the learning domain this translates to a process of interviewing learning domain experts and examining teaching and learning materials.

For our own knowledge acquisition the authors examined the output of the OTM 2004 Workshop on Ontologies, Semantics and E-learning [7] and looked at online course resources (including course notes and syllabuses) and created an initial concept graph. This was then verified with a domain expert in a series of interviews, which resulted in a list of key learning concepts, attributes and relationships.

The next part of the Knowledge life cycle is Modelling. We built our initial ontology in Protégé 2000 [8], an ontology building and knowledge acquisition tool that is frequently used for knowledge modelling. It allows knowledge engineers to focus on modelling without worrying about underlying language and syntax. The model can be saved in various formats including RDF and OWL.

The next step is Knowledge Annotation, this is also termed knowledge binding [9], and depends upon human effort to tag the resources. Generating the instances involves annotating the raw data source using pre-defined ontologies.

Protégé can also be used to instantiate an ontology. It may then be treated as a knowledge base or the instances can be exported from protégé (with an OWL plug-in). The task of ontology instantiation is specialised skill that requires knowledge engineers to translate resource information into the ontology, this is often too complicated for resource providers. For the occasions an annotaion tool would be preferable to allow users to do the annotations themselves.

Once the resources are enriched with semantics, we enter the Knowledge Reuse stage. The knowledge could be resued to assist resource discovery (enrich resources on the Web so that they can be easily identified), process automation (as services have their interface, parameters and effects semantically described, automation becomes possible) or service integration (where simpler services can be combined together to realise more complex customised functionalities). Our intention is to pursue these three objectives, with the first two acting as stepping stones to the third.

\section{Conclusion}

In this paper we have argues that sophisticated semantics can enrich learning resources and enable the paradigm shift from information based content delivery to knowledge based, context-aware collaberative learning services. Ontologies can be used as an improvement over exisitng metadata efforts to bring in the semantics needed for these enriched services and resources.

We have also proposed the use of the Knowledge Life Cycle to manage the key phases in modelling learning semantics. We have described our efforts to follow a life cycle model within the learning domain - namely by performing an acquisition exercise, building a leaning ontology and creating semantic instances in Protégé in order to explore automation and reuse in the future. We believe that a Knowledge Life Cycle model is critical to successfully managing learning and teaching semantics and achieving the goals of resource sharing, collaboration and automation.

\section{Acknowledgements}

Part of this work was funded by The European Commission under the E-Learning Grid Infrastructure project (ELeGI), IST-0002205, Sixth Framework Programme.

\section{References}

1. Judith Rodriguez, Luis Anido, Manuel J. Fernandez, "How can the Web Services Paradigm improve E-learning?" 3rd IEEE International Conference on Advanced Learning Technologies (ICALT'03), July 9-11, Athens, Greece, 2003

2. Toshio Okamoto, Mizue Kayama, "The Learning GRID for Knowledge Building", 4th IEEE International Conference on Advanced Learning Technologies (ICALT'04) August 30 September 01, Joensuu, Finland, 2004

3. Marco Ronchetti, Paramjeet Saini, "Knowledge management in an e-learning system", 4th IEEE International Conference on Advanced Learning Technologies (ICALT'04) August 30 September 01, Joensuu, Finland, 2004 pp. 365-369

4. Bhavani Sridharan, Alexei Tretiakov and Kinshuk, "Application of Ontology to Knowledge Management in Web based Learning", 4th IEEE International Conference on Advanced Learning Technologies (ICALT'04) August 30 September 01, Joensuu, Finland, 2004, pp 663-665

5. Demetrios G. Sampson, Miltiadis D. Lytras, Gerd Wagner and Paloma Diaz (Eds): "Ontologies and the Semantic Web for Elearning", Special Issue of IEE IFETS Journal of Educational Technology \& Society Special: 2004, Vol. 7, Issue 4

6. Guus Schreiber, Hans Akkermans, Anjo Anjewierden, Robert de Hoog, Nigel Shadbolt, Walter Van de Velde and Bob Wielinga, "Knowledge Engineering and Management: The CommonKADS Methodology", MIT Press, Dec 1999

7. OTM 2004 Workshop on Ontologies, Semantics and Elearning (28/10/2004), Larnaca, Cyprus, 2004 http://www.starlab.vub.ac.be/events/OTM04WOSE/

8. Protégé homepage, http://protege.stanford.edu/index.html

9. Tao, F., Cox, S., Chen, L., Shadbolt, N., Xu, F., Puleston, C., Goble, C. and Song, W. 'Towards the Semantic Grid: Enriching Content for Management and Reuse', Delivering eScience, UK e-Science All-hand Conference 2003, Nott, UK. 